\title{
Human $\beta$-defensin 3-combined gold nanoparticles for enhancement of osteogenic differentiation of human periodontal ligament cells in inflammatory microenvironments
}

This article was published in the following Dove Press journal: International Journal of Nanomedicine

\author{
Jing Zhou' \\ Yangheng Zhang' \\ Lingjun $\mathrm{Li}^{\prime}$ \\ Huangmei $\mathrm{Fu}^{2}$ \\ Wenrong Yang ${ }^{2}$ \\ Fuhua Yan'
}

'Nanjing Stomatological Hospital, Medical School of Nanjing University, Nanjing, Jiangsu, People's Republic of China; ${ }^{2}$ School of Life and Environmental Science, Centre for Chemistry and Biotechnology, Deakin University, Geelong, VIC, Australia
Correspondence: Fuhua Yan Nanjing Stomatological Hospital, Medical School of Nanjing University, 30 Zhongyang Road, Nanjing, Jiangsu 210008 , People's Republic of China

Tel +862583620253

Fax +862583620202

Email yanfh@nju.edu.cn

\begin{abstract}
Objective: It is a great challenge to absorb and conduct biophysicochemical interactions at the nano-bio interface. Peptides are emerging as versatile materials whose function can be programmed to perform specific tasks. Peptides combined nanoparticles might be utilized as a new approach of treatment. Human $\beta$-defensin 3 (hBD3), possesses both antimicrobial and proregeneration properties. Gold nanoparticles (AuNPs) have shown promising applications in the field of tissue engineering. However, the coordinating effects of AuNPs and hBD3 on human periodontal ligament cells (hPDLCs) remain unknown. In this study, we systematically investigated whether AuNPs and hBD3 would be able to coordinate and enhance the osteogenic differentiation of hPDLCs in inflammatory microenvironments, and the underlying mechanisms was explored.
\end{abstract}

Methods: hPDLCs were stimulated with E. coli-LPS, hBD3 and AuNPs. Alkaline phosphatase (ALP) and alizarin red S staining were used to observe the effects of hBD3 and AuNPs on the osteogenic differentiation of hPDLCs. Real-time PCR and western blot were performed to evaluate the osteogenic differentiation and $\mathrm{Wnt} / \beta$-catenin signaling pathway related gene and protein expression

Results: In the inflammatory microenvironments stimulated by E. coli-LPS, we found that AuNPs and hBD3 increased the proliferation of hPDLCs slightly. In addition, hBD3-combined AuNPs could significantly enhance ALP activities and mineral deposition in vitro. Meanwhile, we observed that the osteogenic differentiation-related gene and protein expressions of ALP, collagenase-I (COL-1) and runt-related transcription factor 2 (Runx-2) were remarkably upregulated in the presence of hBD3 and AuNPs. Moreover, hBD3-combined AuNPs strongly activated the $\mathrm{Wnt} / \beta$-catenin signaling pathway and upregulated the gene and protein expression of $\beta$-catenin and cyclin D1. Furthermore, hBD3-combined AuNPs induced osteogenesis, which could be reversed by the Wnt/ $\beta$-catenin signaling pathway inhibitor (ICG-001).

Conclusion: The present study demonstrated that hBD3 combined AuNPs could significantly promote the osteogenic differentiation of hPDLCs in inflammatory microenvironments via activating the $\mathrm{Wnt} / \beta$-catenin signaling pathway.

Keywords: hBD3, gold nanoparticles, periodontal ligament cells, osteogenesis, inflammatory microenvironments, Wnt $/ \beta$-catenin signaling

\section{Introduction}

Periodontitis is defined as a bacterially induced chronic inflammatory disease, which compromises the integrity of the tooth-supporting tissues. ${ }^{1}$ The last stage includes 
destruction of periodontal tissue, progressive alveolar bone loss, and eventual tooth loss. ${ }^{2}$ As it is known, the fundamental aims of periodontal therapy are to control periodontal tissue inflammation and to produce regeneration in local destroyed periodontal tissues. ${ }^{3}$ The periodontal tissues include the gingiva, alveolar bone, and periodontal ligament (PDL). The PDL is composed of fibrous tissue that connects the tooth root to the alveolar bone. ${ }^{4}$ PDL cells (PDLCs) have roles in tissue regeneration, which can produce bone-related proteins, express alkaline phosphatase (ALP), and possess mineralization capacity. ${ }^{5}$ Therefore, the PDL system has the high regenerative capacity and potential to give rise to all of the periodontium.

Defensins as one of the antimicrobial peptides, which are subdivided into $\alpha$ - and $\beta$-defensins, play a vital role in the human immune response. ${ }^{6}$ Human $\beta$-defensins can be found in epithelial surfaces, including human $\beta$-defensin $1,2,3$, and 4. Human $\beta$-defensin 3 (hBD3) possesses the particularity that it stays dynamic in different realms of biological activities. ${ }^{7}$ Our previous studies revealed that hBD3 exhibits anti-inflammatory properties both in RAW 264.7 cells $^{8}$ and human umbilical vein endothelial cells, ${ }^{9}$ and it also has a therapeutic effect on systemic inflammation associated with periodontal infections. ${ }^{10}$ In addition, hBD3 enhanced the differentiation of osteoblast-like MG63 cells positively ${ }^{11}$ and played an important role in promoting periodontal regeneration, which might act as a potent regeneration promoter in infectious diseases. ${ }^{12}$

Another material, such as gold nanoparticles (AuNPs), has been widely used for biomedical applications, ${ }^{13-15}$ in particular, in the field of tissue engineering. AuNPs could promote the differentiation of adipase derived stem cells, ${ }^{16}$ mesenchymal stem cells, ${ }^{17}$ MC3T3-E1 cells, ${ }^{18}$ and MG63 cells. ${ }^{19}$ Furthermore, among all the properties, the sizes of nanoparticles are noteworthy. $\mathrm{Li}$ et $\mathrm{al}^{20}$ indicated that 40 and $70 \mathrm{~nm}$ AuNPs could promote the osteogenesis of mesenchymal stem cells when compared with $110 \mathrm{~nm}$ AuNPs. Moreover, Jiang et $\mathrm{al}^{21}$ found that AuNPs on SK-BR-3 cells were most effective when their sizes were between 40 and $50 \mathrm{~nm}$. Meanwhile we also demonstrated that $45 \mathrm{~nm}$ AuNPs could significantly promote the osteogenesis of human PDL progenitor cells when compared with 5 and $13 \mathrm{~nm}$ AuNPs. ${ }^{22}$ Therefore, we chose $45 \mathrm{~nm}$ AuNPs as one of the materials to evaluate the effect on osteogenic differentiation in this study.

The Wnt/ $\beta$-catenin signaling pathway has a pivotal effect on the promotion of osteogenesis and bone formation. ${ }^{23}$ AuNPs could promote osteogenesis of mesenchymal stem cells via the $\mathrm{Wnt} / \beta$-catenin signaling pathway. ${ }^{24} \mathrm{~A}$ previous study also suggested a novel mechanism, Wnt/ $\beta$-catenin signaling, that mediated osteogenic differentiation, which could improve the bone mass and microarchitecture in the aging skeleton. ${ }^{25}$

However, few studies have put the focus on the osteogenic differentiation of human PDLCs (hPDLCs) through the coordination of AuNPs and hBD3 in inflammatory microenvironments; particularly, it is not clear that, when hBD3 combined AuNPs, whether their functions could be more than just a sum of single function. Thus, in this study, we used hBD3-combined AuNPs to enhance and regulate the osteogenic differentiation of hPDLCs under inflammatory microenvironments. In addition, we further investigated the role of $\mathrm{Wnt} / \beta$-catenin signaling pathway in the process of AuNPs and hBD3-mediated osteogenic differentiation.

\section{Materials and methods Cell culture and reagents}

hPDLCs (ScienCell Research Laboratories, Carlsbad, CA, USA) were cultured in growth low-glucose DMEM (Gibco cell culture; Thermo Fisher Scientific, Waltham, MA, USA) supplemented with $1 \%$ penicillin/streptomycin (HyClone, Logan, UT, USA) and 10\% fetal bovine serum (ScienCell Research Laboratories) at $37^{\circ} \mathrm{C}$ in $5 \% \mathrm{CO}_{2}$. Cells were used between passages 2 and 6 in subsequent experiments. After cell confluency, an osteogenic-inducing medium supplemented with $10 \mathrm{mM} \beta$-glycerophosphate (Sigma-Aldrich Co., St Louis, MO, USA), $0.1 \mu \mathrm{M}$ dexamethasone (Sigma-Aldrich Co.) and $50 \mu \mathrm{g} / \mathrm{mL}$ of L-ascorbic acid (Sigma-Aldrich Co.) was replaced.

hBD3 was commercially available from Peprotech (Rocky Hill, NJ, USA), and the concentration was used according to our published data. ${ }^{8}$ AuNPs with diameter $45 \mathrm{~nm}$ were synthesized by adopting chemical reduction method based on our previous report. ${ }^{26}$ Lipopolysaccharides (LPS) (Escherichia coli 055:B5, L2880) was obtained from Sigma-Aldrich Co. Wnt/ $\beta$-catenin signaling pathway inhibitor ICG-001 was purchased from Selleck Chemicals (Houston, TX, USA).

\section{Cell viability assay}

The cell counting kit-8 (CCK-8; Dojindo Molecular Technologies, Tokyo, Japan) was used to analyze the cell viability of hPDLCs. The cells were grown in 96-well plates at a density of $5.0 \times 10^{3}$ cells/well. After cell confluency, hBD3 $(5 \mu \mathrm{g} / \mathrm{mL}), 45 \mathrm{~nm}$ AuNPs $(10 \mu \mathrm{M})$, and E. coli-LPS $(1 \mu \mathrm{g} / \mathrm{mL})$ were added. After 7 days of incubation, every 
well was added $10 \mu \mathrm{L}$ of CCK-8 reagent and incubated for 4 h. The SpectraMax M3 Microplate Spectrophotometer (Molecular Devices, Sunnyvale, CA, USA) was used to measure the optical density of each well at the absorbance of $450 \mathrm{~nm}$.

\section{Transmission electron microscopy (TEM)}

TEM was used to examine the uptake of AuNPs. hPDLCs were grown in six-well plates at a density of $2.0 \times 10^{5}$ cells/well. After cell confluency, hBD3 (5 $\mu \mathrm{g} / \mathrm{mL}), 45 \mathrm{~nm}$ AuNPs $(10 \mu \mathrm{M})$, and E. coli-LPS $(1 \mu \mathrm{g} / \mathrm{mL})$ were added. After 7 days of incubation, fixation, embedding, cutting into ultrathin sections, analysis with TEM (HT7700; Hitachi Ltd., Tokyo, Japan) were carried out.

\section{ALP activity and staining}

hPDLCs were grown in 24-well plates at a density of $5.0 \times 10^{4}$ cells/well. After cell confluency, hBD3 (5 $\mu \mathrm{g} / \mathrm{mL}), 45 \mathrm{~nm}$ AuNPs $(10 \mu \mathrm{M})$ and $E$. coli-LPS $(1 \mu \mathrm{g} / \mathrm{mL})$ were added. ALP activity assay and ALP staining were performed on day 7 .

For ALP staining, an ALP staining kit (Beyotime Institute of Biotechnology, Shanghai, China) was used after fixing the cells with 4\% paraformaldehyde. Then, PBS was used to stop the staining reaction. Inverted optical microscopy (IMT-2; Olympus Corporation, Tokyo, Japan) was used to observe the ALP staining.

Alkaline Phosphatase Assay Kit (ab83369; Abcam, Cambridge, MA, USA) was applied to analyze the ALP activity. The cells were lysed and centrifuged, later then the supernatant from each sample was incubated with p-nitrophenyl phosphate solution in 96-well plates at $25^{\circ} \mathrm{C}$ for $60 \mathrm{~min}$ in the dark. Subsequently, stop solution was added to stop the reaction and the results were measured at $405 \mathrm{~nm}$ using a SpectraMax M3 Microplate Spectrophotometer.

\section{Alizarin red $S$ (ARS) staining}

hPDLCs were grown in 24-well plates at a density of $5.0 \times 10^{4}$ cells/well. After cell confluency, hBD3 (5 $\mu \mathrm{g} / \mathrm{mL}), 45 \mathrm{~nm}$
AuNPs $(10 \mu \mathrm{M})$ and $E$. coli-LPS $(1 \mu \mathrm{g} / \mathrm{mL})$ were added. ARS staining and quantitation assay were performed on day 21 .

For ARS staining, 2\% ARS staining solution (SigmaAldrich Co.) was used to stain the cells for 10 min after fixing with 4\% paraformaldehyde and was then examined using an inverted fluorescence microscope (IMT-2).

For ARS quantitation, 10\% (w/v) cetylpyridinium chloride (Sigma-Aldrich Co.) was applied after staining. The absorbance was measured by using a SpectraMax M3 Microplate Spectrophotometer at $562 \mathrm{~nm}$.

\section{Quantitative real-time polymerase chain reaction (PCR)}

hPDLCs were grown in six-well plates at a density of $2.0 \times 10^{5}$ cells/well. After cell confluency, hBD3 $(5 \mu \mathrm{g} / \mathrm{mL})$, $45 \mathrm{~nm}$ AuNPs $(10 \mu \mathrm{M})$ and $E$. coli-LPS $(1 \mu \mathrm{g} / \mathrm{mL})$ were added. After 7 days of incubation, the RNA was isolated using the TRNzol Reagent (Tiangen, Beijing, China) and reverse transcription was performed using the PrimeScript ${ }^{\mathrm{TM}}$ RT Reagent Kit (Takara Bio, Otsu, Japan) according to the manufacturer's instructions. Each primer was designed for real-time PCR, as listed in Table 1 , and the results were analyzed using the $2^{-\Delta \Delta C t}$ method.

\section{Western blot analysis}

hPDLCs were grown in six-well plates at a density of $2.0 \times 10^{5}$ cells/well. After cell confluency, hBD3 $(5 \mu \mathrm{g} / \mathrm{mL})$, $45 \mathrm{~nm}$ AuNPs $(10 \mu \mathrm{M})$, and E. coli-LPS $(1 \mu \mathrm{g} / \mathrm{mL})$ were added. After 7 days of incubation, cells were lysed in the radioimmunoprecipitation assay lysis buffer (Beyotime Institute of Biotechnology). Protein extracts were subjected to sodium dodecyl sulfate polyacrylamide gel electrophoresis and then transferred onto polyvinylidene fluoride membranes. A total of 5\% skim milk in tris buffered saline were used to block the membranes for $2 \mathrm{~h}$ before incubating overnight with specific primary antibodies, ALP and collagenase-I (COL-1) from Abcam and runt-related transcription factor 2 (Runx-2), $\beta$-catenin, and cyclin D1 from Cell Signaling Technology Inc. (Danvers, MA, USA). $\beta$-Actin (Cell Signaling Technology Inc.)

Table I Primers for quantitative real-time PCR analysis

\begin{tabular}{|c|c|c|c|}
\hline $\begin{array}{l}\text { Primer } \\
\text { name }\end{array}$ & Forward primer sequence $\left(5^{\prime}-3^{\prime}\right)$ & Reverse primer sequence $\left(5^{\prime}-3^{\prime}\right)$ & $\begin{array}{l}\text { Accession } \\
\text { number }\end{array}$ \\
\hline$\overline{A L P}$ & CCGTGGCAACTCTATCTTTGG & GCCATACAGGATGGCAGTGA & NM000478 \\
\hline COL-I & CTGCAAGAACAGCATTGCAT & GGCGTGATGGCTTATTTGTT & NM000089 \\
\hline Runx-2 & GGAGTGGACGAGGCAAGAGTTT & AGCTTCTGTCTGTGCCTTCTGG & XM0II5I4966.2 \\
\hline Cyclin DI & TGATGCTGGGCACTTCATCTG & TCCAATCATCCCGAATGAGAGTC & NM053056.2 \\
\hline GAPDH & CGCTCTCTGCTCСTCСTGTT & CCATGGTGTCTGAGCGATGT & NM002046.5 \\
\hline
\end{tabular}

Abbreviations: ALP, alkaline phosphatase; COL-I, collagenase-I; PCR, polymerase chain reaction; Runx-2, runt-related transcription factor 2; GAPDH, glyceraldehyde 3-phosphate dehydrogenase. 
was used as an internal control. Tris-buffered saline containing $0.1 \%$ Tween 20 was applied to wash the blots and then incubated with secondary antibodies for $2 \mathrm{~h}$. Immunoreactive bands were detected with a chemiluminescence kit, and imaging system (LAS4000M; GE Healthcare Bio-Sciences Corp., Piscataway, NJ, USA) was used to capture the pictures.

\section{Statistical analysis}

All data were shown by the mean \pm SD. Comparisons between different treatments were performed with a one-way analysis of variance with Bonferroni's post hoc test using the statistical GraphPad Prism vision 6.0 software. Statistical significance was set at $P$-values $<0.05$.

\section{Results}

\section{Effects of hBD3 on viability, ALP activity, and osteogenic gene expression of hPDLCs stimulated with E. coli-LPS}

First, we evaluated the viability of hPDLCs treated with hBD3 $(5 \mu \mathrm{g} / \mathrm{mL})$ and $E$. coli-LPS $(1 \mu \mathrm{g} / \mathrm{mL})$ using the
CCK-8 assay at day 7. As shown in Figure 1A, E. coli-LPS at a concentration of $1 \mu \mathrm{g} / \mathrm{mL}$ had little effect on the proliferation of hPDLCs. In addition, treatment with hBD3 at $5 \mu \mathrm{g} / \mathrm{mL}$ markedly increased the cell viability. The results also suggested that hBD3 had no influence on the viability of hPDLCs.

Next, we examined the effects of hBD3 on the osteogenesis of hPDLCs using an ALP staining and ALP activity assay. On day 7, hBD3 increased ALP activity stimulated by E. coli-LPS (Figure 1B). The ALP activity assay also revealed the same tendency as the ALP staining of 7 days (Figure 1C).

Third, the effects of hBD3 on ALP, COL-1, and Runx-2 mRNA expression levels were determined by real-time PCR on day 7. As shown in Figure 1D, E. coli-LPS inhibited ALP mRNA expression level, while hBD3 significantly accelerated mRNA expression level stimulated by E. coli-LPS. Similarly, the mRNA expression of COL-1 and Runx-2 was significantly increased in the hBD3 $+E$. coli-LPS group than in the E. coli-LPS group. Based on the results of mRNA

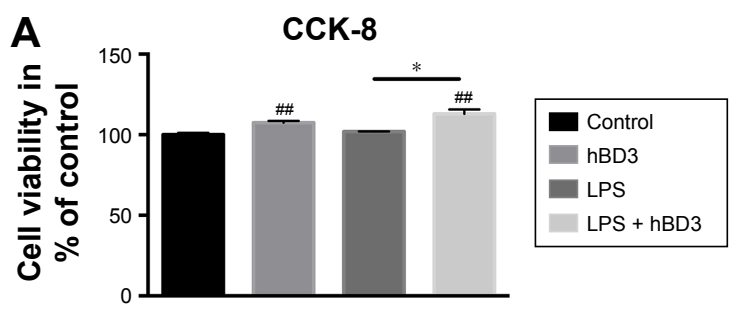

\section{$B_{\text {hBD3 }}$ E. coli-LPS}
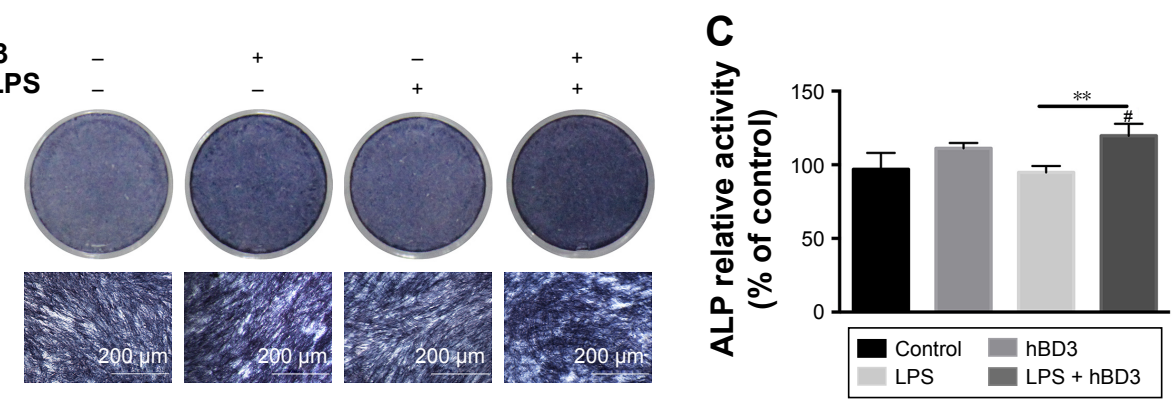

D
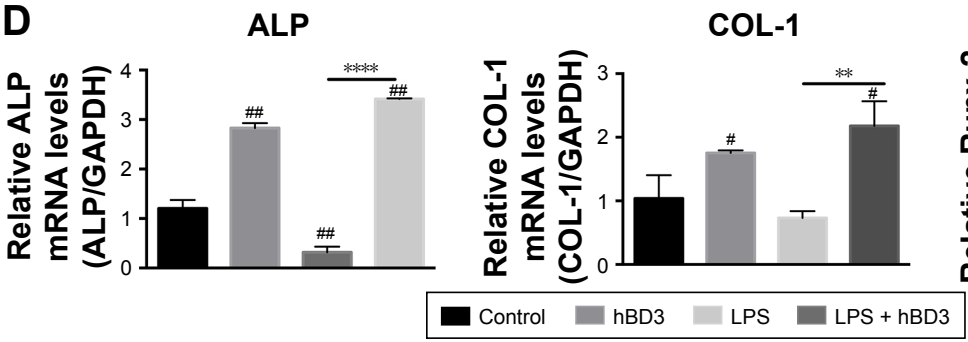

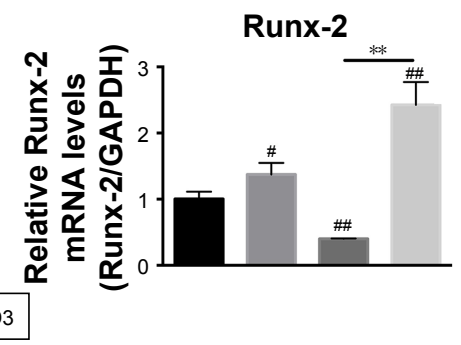

Figure I Effects of hBD3 on the cell viability and osteogenic differentiation of hPDLCs in inflammatory microenvironments.

Notes: hPDLCs were treated with hBD3 $(5 \mu \mathrm{g} / \mathrm{mL})$ and $E$. coli-LPS $(I \mu \mathrm{g} / \mathrm{mL})$. (A) The cell viability of hPDLCs was analyzed with a CCK-8 assay on day 7 . (B) ALP staining and (C) ALP activity of hPDLCs after hBD3 treatment. (D) ALP, COL-I, and Runx-2 mRNA expressions on day 7 analyzed by real-time PCR. ${ }^{2}<0.05$ and $\# P<0.01$ compared with the control group; $* P<0.05, * * P<0.01$, and $* * * * P<0.000$ I.

Abbreviations: ALP, alkaline phosphatase; CCK-8, cell counting kit-8; COL-I, collagenase-I; E. coli, Escherichia coli; hBD3, human $\beta$-defensin 3; hPDLCs, human periodontal ligament cells; LPS, lipopolysaccharides; PCR, polymerase chain reaction; Runx-2, runt-related transcription factor 2. 
expression, hBD3 elevated the expression of osteogenic markers stimulated by E. coli-LPS.

\section{Effects of hBD3-combined AuNPs on hPDLCs' viability stimulated with E. coli- LPS and uptake of AuNPs in hPDLCs}

As shown in Figure 2A, hBD3 $(5 \mu \mathrm{g} / \mathrm{mL}), 45 \mathrm{~nm}$ AuNPs $(10 \mu \mathrm{M})$, and E. coli-LPS $(1 \mu \mathrm{g} / \mathrm{mL})$ exhibited no significant cytotoxicity to hPDLCs. On day 7, the cell viability of hPDLCs had a slight increase in the hBD3, AuNPs, and E. coli-LPS group. The result indicated a good biocompatibility of hBD3, $45 \mathrm{~nm}$ AuNPs, and E. coli-LPS when the concentration was at $5 \mu \mathrm{g} / \mathrm{mL}, 10 \mu \mathrm{M}$, and $1 \mu \mathrm{g} / \mathrm{mL}$, respectively. Therefore, hBD3 $(5 \mu \mathrm{g} / \mathrm{mL}), 45 \mathrm{~nm}$ AuNPs $(10 \mu \mathrm{M})$, and E. coli-LPS $(1 \mu \mathrm{g} / \mathrm{mL})$ were used in the following experiments.

The TEM showed the uptake of AuNPs. Figure 2B reveals that $45 \mathrm{~nm}$ AuNPs with a spherical shape were internalized and one or two AuNPs clustered together. In addition, AuNPs were localized in intracellular vesicles among all the AuNPs treated groups compared with controls.

\section{Effects of hBD3-combined AuNPs on ALP activity and mineralization of hPDLCs stimulated with E. coli-LPS}

First, the effects of hBD3-combined AuNPs on the early osteogenic differentiation of hPDLCs prompted by E. coliLPS was assessed by staining for ALP on day 7 (Figure 3A, ALP). The ALP activity of hPDLCs treated with hBD3, AuNPs, and E. coli-LPS was significantly increased and was also higher than that in the hBD3+E. coli-LPS group. An ALP activity assay on day 7 exhibited similar tendency with ALP staining (Figure 3B).

Next, formation of mineralized nodules (Figure 3A, ARS) and calcium deposition (Figure 3C) were used to evaluate the effects of hBD3 and AuNPs on the late osteogenesis of
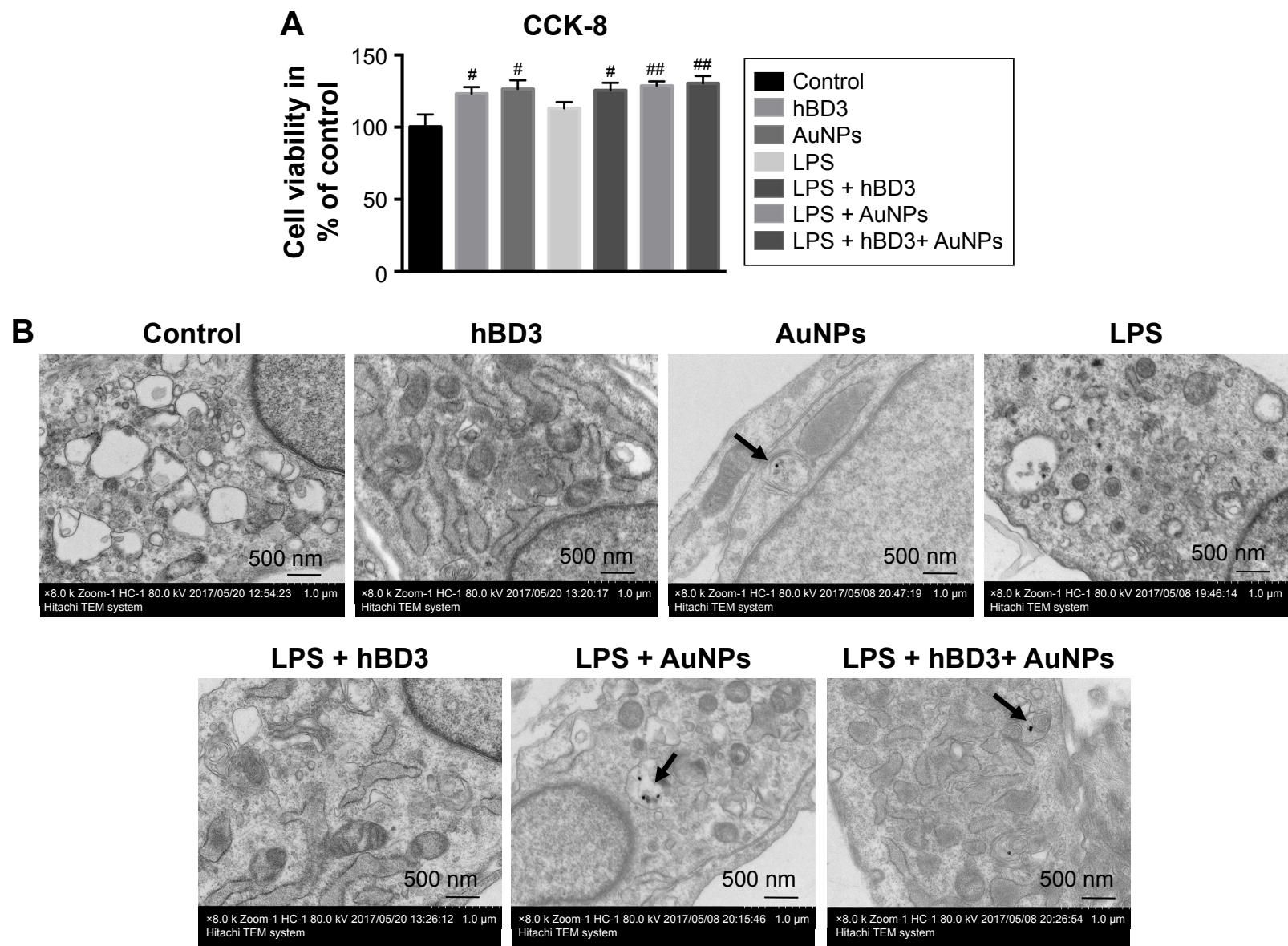

Figure 2 Effects of hBD3-combined AuNPs on cell viability of hPDLCs and cellular uptake of AuNPs in inflammatory microenvironments.

Notes: hPDLCs were treated with hBD3 $(5 \mu \mathrm{g} / \mathrm{mL})$, AuNPs $(45 \mathrm{~nm}, 10 \mu \mathrm{M})$, and E. coli-LPS $(\mathrm{I} \mu \mathrm{g} / \mathrm{mL})$. (A) The biocompatibility of hPDLCs measured by CCK-8 on day 7 . (B) TEM images of uptake of AuNPs on day 7. Arrows indicate internalized AuNPs. ${ }^{\#}<<0.05$ and ${ }^{\# P}<0.01$ compared with the control group.

Abbreviations: AuNPs, gold nanoparticles; CCK-8, cell counting kit-8; E. coli, Escherichia coli; hBD3, human $\beta$-defensin 3; hPDLCs, human periodontal ligament cells; LPS, lipopolysaccharides; TEM, transmission electron microscopy. 


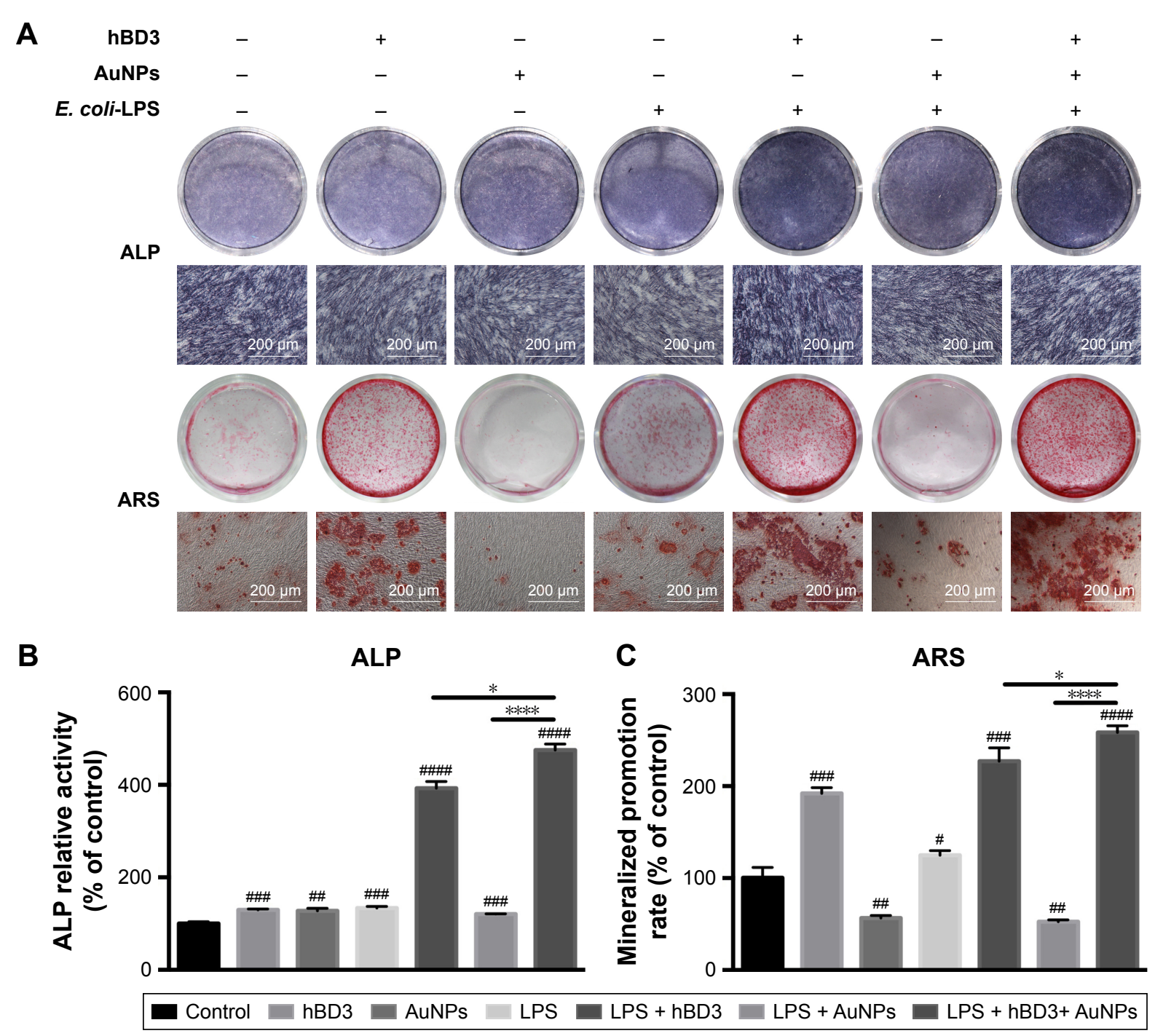

Figure 3 Effects of hBD3-combined AuNPs on the ALP activity and calcium deposition of hPDLCs in inflammatory microenvironments.

Notes: hPDLCs were treated with hBD3 $(5 \mu \mathrm{g} / \mathrm{mL})$, AuNPs $(45 \mathrm{~nm}, 10 \mu \mathrm{M})$, and E. coli-LPS $(1 \mu \mathrm{g} / \mathrm{mL})$. (A) ALP staining on day 7 and calcium deposition staining with ARS

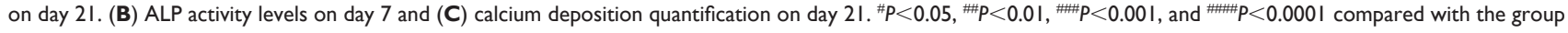
control; $* P<0.05$ and $* * * * P<0.0001$.

Abbreviations: ALP, alkaline phosphatase; ARS, alizarin red S; AuNPs, gold nanoparticles; E. coli, Escherichia coli; hBD3, human $\beta$-defensin 3; hPDLCs, human periodontal ligament cells; LPS, lipopolysaccharides.

hPDLCs. After 21 days of osteogenic differentiation, mineralized nodules were visualized by ARS staining. As shown in Figure 3A (ARS), the stain color grew deeper and thicker with coordination of hBD3, AuNPs, and E. coli-LPS, indicating that the amount of accumulated mineral matrix deposition was increased. We also quantified the calcium content in Figure 3C and compared it with the control group; ARS deposition was remarkably increased in the presence of hBD3, AuNPs, and E. coli-LPS. Notably, cells treated with hBD3, AuNPs, and E. coli-LPS produced more calcium deposition than that in the hBD3+E. coli-LPS group, which was similar to the ALP activity assay.

\section{Effects of hBD3-combined AuNPs on the} hPDLCs' osteogenic gene and protein expressions stimulated with E. coli-LPS

The effects of hBD3-combined AuNPs prompted with E. coli-LPS on ALP, COL-1, and Runx-2 mRNA expression levels of hPDLCs were determined by real-time PCR on day 7. As shown in Figure 4A, ALP mRNA expression was increased in hPDLCs cultured with hBD3+AuNPs + E. coliLPS on day 7 and was significantly upregulated compared to the hBD3+E. coli-LPS group and the AuNPs + E. coli-LPS group. The COL-1 mRNA level in the hBD3+ AuNPs + E. coli-LPS group was remarkably increased compared to the control, and the mRNA level was approximately twofold 
A

ALP

COL-1

Runx-2
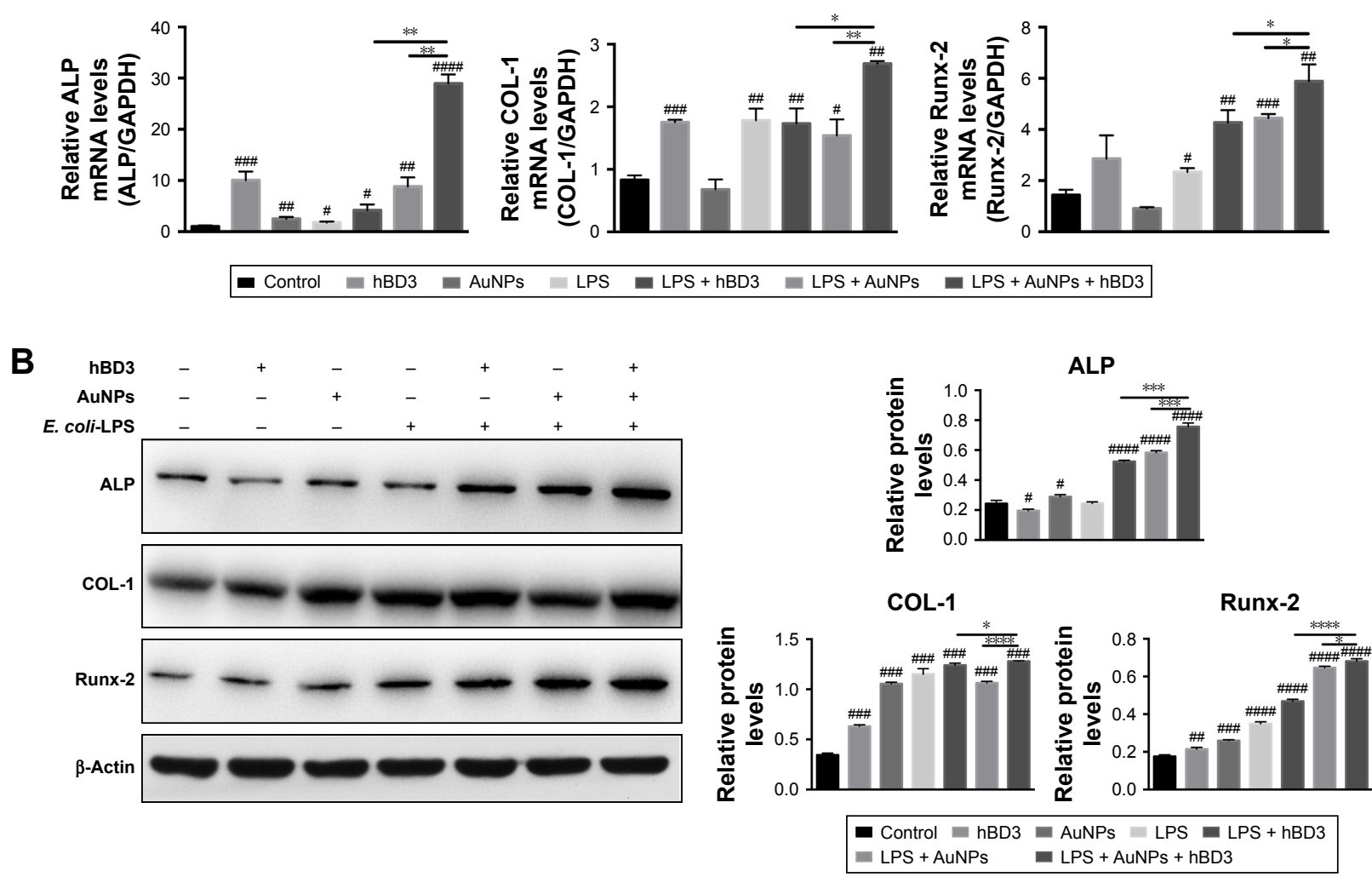

Figure 4 Effects of hBD3-combined AuNPs on osteogenic gene and protein expressions of hPDLCs in inflammatory microenvironments.

Notes: hPDLCs were treated with hBD3 $(5 \mu \mathrm{g} / \mathrm{mL})$, AuNPs $(45 \mathrm{~nm}, 10 \mu \mathrm{M})$, and E. coli-LPS $(1 \mu \mathrm{g} / \mathrm{mL})$. (A) The mRNA expression levels of ALP, COL-I, and Runx-2 analyzed by real-time PCR on day 7. (B) The protein expression levels of ALP, COL-I, and Runx-2 analyzed by Western blot on day 7. ${ }^{P P}<0.05,{ }^{\# P}<0.01$, ${ }^{\#} P<0.001$, and \# $P<0.000$ I compared with the control group; $* P<0.05$, $* * P<0.0$ I, $* * * P<0.001$, and $* * * * * P<0.000$ I.

Abbreviations: ALP, alkaline phosphatase; AuNPs, gold nanoparticles; COL-I, collagenase-l; E. coli, Escherichia coli; hBD3, human $\beta$-defensin 3; hPDLCs, human periodontal ligament cells; LPS, lipopolysaccharides; PCR, polymerase chain reaction; Runx-2, runt-related transcription factor 2.

higher than that of the AuNPs $+E$. coli-LPS group on day 7 . Similarly, the mRNA expression of Runx-2 was significantly increased in the hBD3+ AuNPs $+E$. coli-LPS group compared to the control, hBD3+E. coli-LPS, and AuNPs + E. coli-LPS groups.

Furthermore, Western blot was used to analyze the protein expression levels of ALP, COL-1, and Runx-2 on day 7 . Figure $4 \mathrm{~B}$ reflects that all three protein expression levels shared the similar tendency with the mRNA results; particularly, it was worth noting that the hBD3+ AuNPs + E. coli-LPS group showed significant upregulation in all three osteogenic markers.

\section{Effects of hBD3-combined AuNPs on osteogenic differentiation stimulated with E. coli-LPS on Wnt/ $\beta$-catenin signaling pathway}

The effects of $\mathrm{Wnt} / \beta$-catenin signaling pathway on the osteogenic differentiation of hPDLCs promoted by hBD3 combined AuNPs were studied on day 7. The mRNA level of the Wnt/ $\beta$-catenin pathway target gene cyclin D1 was markedly regulated in the presence of hBD3+ AuNPs + E. coli-LPS (Figure 5A). According to the results of protein expression levels (Figure 5B), Wnt/ $\beta$-catenin pathwayrelated regulator $\beta$-catenin was highest in the AuNPs + E. coli-LPS group than that in the hBD3+E. coli-LPS and AuNPs + hBD3 $+E$. coli-LPS groups, while the cyclin D1 was only upregulated in the hBD3 $+E$. coli-LPS group. To confirm whether the $\mathrm{Wnt} / \beta$-catenin signaling pathway was involved in the osteogenesis of hPDLCs, the Wnt/ $\beta$-catenin inhibitor ICG-001 $(10 \mu \mathrm{M})$ was added to the hBD3+ AuNPs + E. coli-LPS-treated hPDLCs and cultured for 7 days. Then, we found that the mRNA expression of cyclin D1 was reduced significantly compared to the hBD3+ AuNPs + E. coli-LPS-treated hPDLCs (Figure 5C). In addition, the cells stimulated with ICG-001 decreased the protein expression of the Wnt pathway-related regulators $\beta$-catenin and cyclin D1 (Figure 5D). 


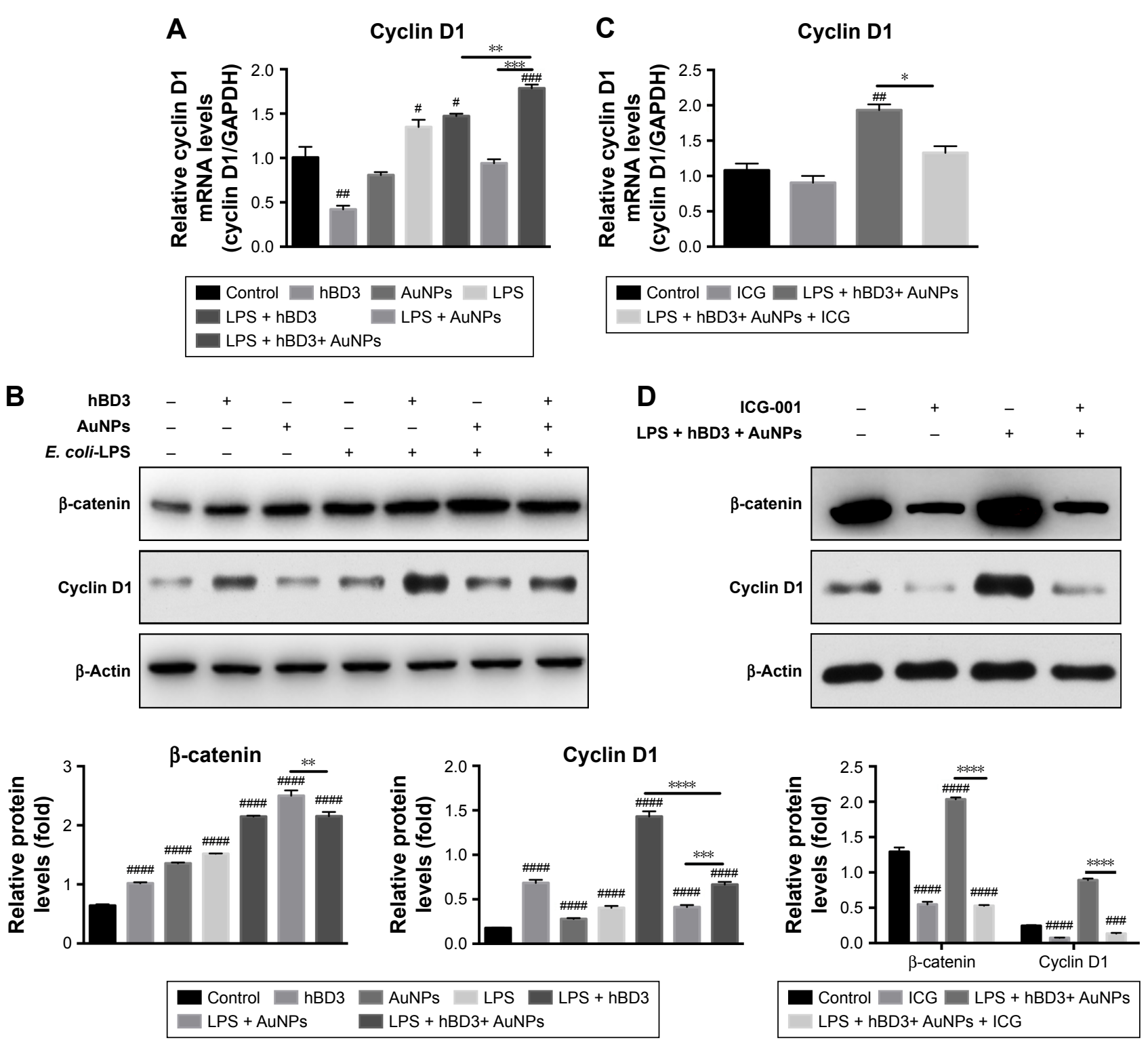

C Cyclin D1

Figure 5 Effects of Wnt/B-catenin signaling pathway on osteogenic differentiation of hPDLCs induced by hBD3-combined AuNPs in inflammatory microenvironments. Notes: hPDLCs were treated with hBD3 $(5 \mu \mathrm{g} / \mathrm{mL})$, AuNPs $(45 \mathrm{~nm}, 10 \mu \mathrm{M})$, E. coli-LPS $(1 \mu \mathrm{g} / \mathrm{mL})$ and ICG-00I $(10 \mu \mathrm{M})$. (A) The Wnt/B-catenin pathway target gene cyclin DI mRNA expression on day 7 analyzed by real-time PCR. (B) $\beta$-Catenin and cyclin DI protein expressions on day 7 analyzed by Western blot. (C) ICG-00I blocked the

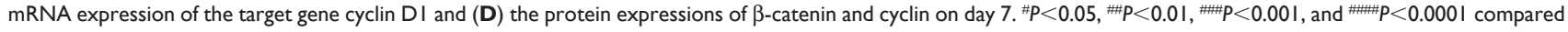
with the control group; $* P<0.05, * * P<0.01, * * * P<0.001$, and $* * * * P<0.0001$.

Abbreviations: AuNPs, gold nanoparticles; E. coli, Escherichia coli; hBD3, human $\beta$-defensin 3; hPDLCs, human periodontal ligament cells; LPS, lipopolysaccharides; PCR, polymerase chain reaction.

\section{Effects of Wnt/ $\beta$-catenin signaling} pathway inhibition on hBD3-combined AuNPs on osteogenic differentiation stimulated with E. coli-LPS

Subsequently, we found that ICG-001 downregulated the hBD3+ AuNPs + E. coli-LPS-stimulated ALP activity and calcium deposition of the hPDLCs (Figure 6A). The ALP activity assay and calcium content (Figure 6B) revealed the similar tendency. Moreover, real-time PCR (Figure 6C) and
Western blot (Figure 6D) showed that the effects of hBD3+ AuNPs $+E$. coli-LPS on mRNA and protein expression levels of ALP, COL-1, and Runx-2 could reverse by the stimulation with ICG-001.

\section{Discussion}

hPDLCs consist of fibroblasts, osteoblasts, cementoblasts, and mesenchymal stem cells, which are capable of differentiating into osteoblast-like cells to achieve periodontal regeneration and repair. ${ }^{27}$ There are several emerging approaches to promote 
A



B

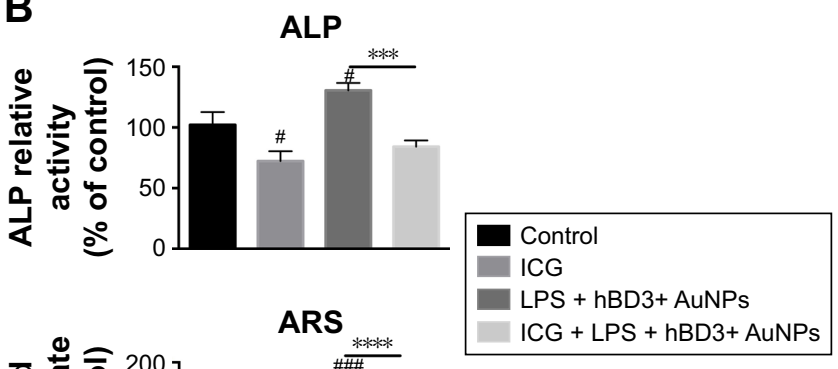

C

ALP
COL-1
Runx-2
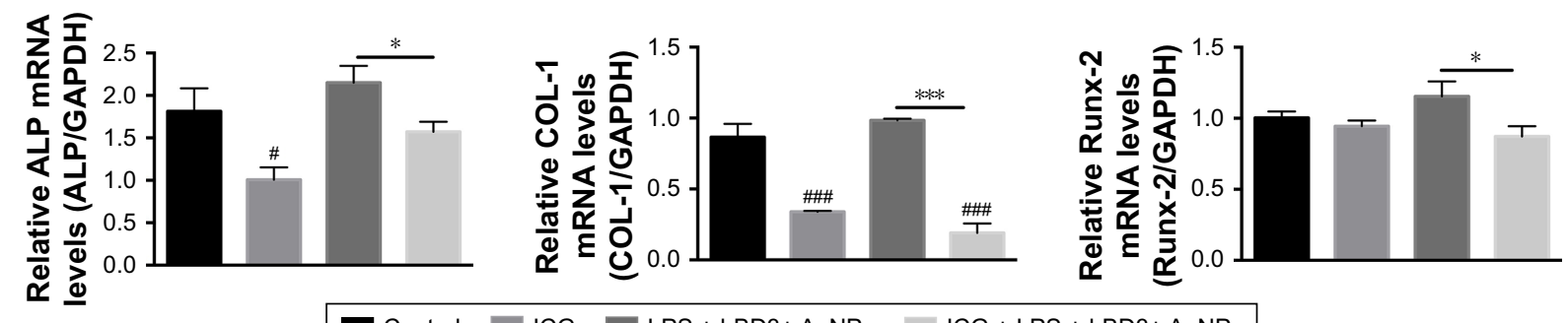

Control ICG LPS + hBD3+ AuNPs ICG + LPS + hBD3+ AuNPs

D
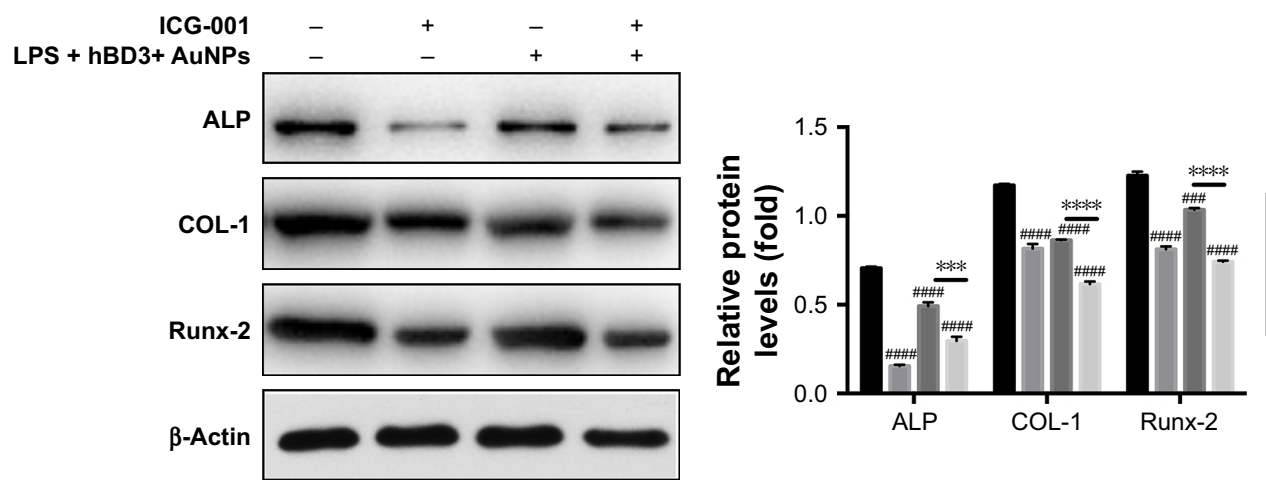

Control
ICG
LPS + hBD3+ AuNPs
ICG + LPS + hBD3+ AuNPs

Figure 6 Effects of ICG-00I on osteogenic differentiation of hPDLCs induced by hBD3-combined AuNPs in inflammatory microenvironments

Notes: hPDLCs were treated with hBD3 $(5 \mu \mathrm{g} / \mathrm{mL})$, AuNPs $(45 \mathrm{~nm}, 10 \mu \mathrm{M})$, E. coli-LPS $(1 \mu \mathrm{g} / \mathrm{mL})$ and ICG-00I (I0 $\mu \mathrm{M})$. (A) ALP staining on day 7 and mineralized nodules staining with ARS on day $2 I$ and (B) ALP activity levels on day 7 and mineralized nodules activity levels on day $2 I$. (C) ALP, COL-I, and Runx-2 mRNA expressions on day 7

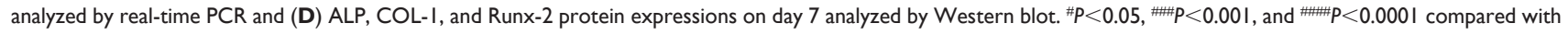
the control group; $* P<0.05$, $* * * P<0.001$, and $* * * * P<0.0001$.

Abbreviations: ALP, alkaline phosphatase; ARS, alizarin red S; AuNPs, gold nanoparticles; COL-I, collagenase-I; E. coli, Escherichia coli; hBD3, human $\beta$-defensin 3; hPDLCs, human periodontal ligament cells; PCR, polymerase chain reaction; Runx-2, runt-related transcription factor 2.

osteogenic cell differentiation, ${ }^{28,29}$ among which, AuNPs and hBD3 might be promising materials for periodontal bone regeneration. However, very few approaches combined antimicrobial with proregenerative properties. Since the capacity for tissue regeneration in a chronic inflammatory microenvironments cannot be inferred, ${ }^{30}$ thus the importance of the properties cannot be denied in the therapeutic process. What is more, the inflammatory microenvironments could be induced by several stimulations, including LPS and tumor necrosis factor-alpha (TNF- $\alpha$ ). TNF- $\alpha$, a group of proinflammatory cytokine, could trigger several signaling cascades, especially the nuclear factor $\mathrm{\kappa B}(\mathrm{NF}-\mathrm{\kappa B})$, c-Jun $\mathrm{NH}_{2}$-terminal kinase (JNK), ${ }^{31}$ and mitogen-activated protein kinases (MAPK), ${ }^{32}$ and eventually induced transcription of inflammatory mediators, while LPS was considered as a potential inducer of proinflammatory cytokines and chemokines, 
which interacted with Toll-like receptors (TLRs). Among these receptors, TLR2 and TLR4 might be related in the progression of periodontitis. ${ }^{33}$ In addition, LPS could increase the TNF- $\alpha$ activity by epithelial cells and was associated with other effects on alveolar bone loss. ${ }^{34}$ Hence treatment with $E$. coli-LPS was used here to mimic the inflammatory microenvironments.

Accordingly, we investigated whether AuNPs and hBD3 could coordinate and promote osteogenesis of hPDLCs in inflammatory microenvironments. The results indicated that hBD3-combined AuNPs could significantly enhance the osteogenic differentiation of hPDLCs under inflammatory microenvironments stimulated by $E$. coli-LPS.

Initially, we evaluated the biotoxicity of AuNPs, hBD3, and $E$. coli-LPS. The CCK-8 assay indicated that $45 \mathrm{~nm}$ AuNPs, hBD3, and E. coli-LPS were safe for hPDLCs in the concentrations of $10 \mu \mathrm{M}, 5 \mu \mathrm{g} / \mathrm{mL}$, and $1 \mu \mathrm{g} / \mathrm{mL}$, respectively. Furthermore, we observed that AuNPs and hBD3 slightly increased the viability of hPDLCs with the stimulation of $E$. coli-LPS, which was in agreement with previous reports. ${ }^{9,22}$

In addition to the biotoxicity, cellular uptake was another essential component in the cellular response to AuNPs. In this study, $45 \mathrm{~nm}$ AuNPs were internalized according to observations of one or two spherical shapes clustered together. Most of them were found in intracellular vesicles. According to the properties of electron dense, the TEM was easy to distinguish AuNPs from other cellular components. ${ }^{35}$ It was found that because of the "wrapping effect," $50 \mathrm{~nm}$ AuNPs were enclosed by cellular membrane at higher rates. ${ }^{36}$ In the TEM images, we could observe that AuNPs were able to enter into the vesicles of cells but rarely enter into the nucleus, which was similar to the previous finding. ${ }^{37}$

ALP was a key component of bone matrix vesicles, which appeared during the early phase of osteoblast differentiation and could accelerate the mineralization of the bone matrix. ${ }^{38}$ The late stage of osteogenesis differentiation was represented by the expression of extracellular matrix mineralization and calcium deposition. In our study, we discovered that the AuNPs + hBD3 $+E$. coli-LPS group had the highest level of ALP staining and activity among all groups and the hBD3+ E. coli-LPS group also demonstrated higher levels than the other groups. Even though the ARS deposition was reduced in the AuNPs and AuNPs $+E$. coli-LPS groups, which might be the reasons that AuNPs alone had the possibility of inhibiting the calcium deposition, but the staining and the calcium content quantification revealed that the AuNPs + hBD3+E. coli-LPS group could remarkably promote mineral matrix deposition. Consequently, all the results showed that the AuNPs + hBD3 $+E$. coli-LPS group especially lead to high osteoblast differentiation.

For a more analytical approach, we evaluated the mRNA and protein expressions of ALP, COL-1, and Runx-2 with real-time PCR and Western blot. Runx-2 was a master regulator of osteoblast differentiation ${ }^{39}$ that upregulated other osteoblast marker genes, such as COL-1 at earlier stages and ALP at later stages of differentiation. ${ }^{40} \mathrm{COL}-1$ could be found in the organic bone matrix with abundant protein $^{41}$ and ALP played the role as a marker, which related to the differentiation of preosteoblasts and osteoblasts. ${ }^{42}$ At day 7 for ALP osteogenic marker, the AuNPs + hBD3 $+E$. coli-LPS group showed the highest levels of expression and the group hBD3+ E. coli-LPS also demonstrated higher levels of COL-1 and Runx-2 than other groups, which were in accordance with the ALP activity and calcium deposition results.

Notably, according to our results, the AuNPs + hBD3+ $E$. coli-LPS group had a significant effect on osteogenic differentiation than the AuNPs $+E$. coli-LPS and hBD3+ E. coli-LPS groups. On one hand, many researchers have reported that AuNPs could promote the mineralization. . $^{4344}$ There was a hypothesis that AuNPs were similar to a crystal nucleus, which resembled the hydroxyapatite crystal in bone. ${ }^{45}$ In addition, it had been reported that the Wnt/ $\beta$-catenin signaling pathway could promote the osteogenesis of chitosan-conjugated AuNPs. ${ }^{24}$ On the other hand, there was a report showing that hBD3 also affected the proliferation and differentiation of eukaryotic cells. ${ }^{46,47}$ It also had been reported that because defensins had similar structures with epidermal growth factor (EGF), hBD3 might influence cell proliferation through the EGF signaling pathway. ${ }^{11}$ Another study demonstrated that hBD3 induces functional maturation of immature human langerhans cell like dendritic cells, which were mediated in part by NF- $\mathrm{KB}$ activation. ${ }^{48}$ However, there were few studies related to the $\mathrm{Wnt} / \beta$-catenin signaling pathway that mediated osteogenesis of hBD3. Therefore, we hypothesized that under inflammatory microenvironments, the $\mathrm{Wnt} / \beta$-catenin signaling pathway played a role in the osteogenic differentiation of hPDLCs stimulated by AuNPs coordinated with hBD3.

In the present study, the mRNA level of cyclin D1 was enhanced after administrating hBD3 and AuNPs, indicating that the $\mathrm{Wnt} / \beta$-catenin signaling pathway had an effect on the osteogenesis of hPDLCs promoted by hBD3-combined AuNPs. Although the protein levels of $\beta$-catenin and cyclin D1 were upregulated by the stimulation of AuNPs, hBD3, and E. coli-LPS, the AuNPs + E. coli-LPS group proved to be the highest expression level of $\beta$-catenin and the hBD3+E. coli-LPS group showed the highest 
expression level of cyclin D1, which had slight differences compared with other results and needed to be further studied. The effects of the Wnt/ $\beta$-catenin signaling pathway on osteogenic differentiation and bone formation had been universally reported. A previous study demonstrated that the Wnt/ $\beta$-catenin signaling pathway activated by mechanical loading and $\beta$-catenin serves as an effector in PDLCs. ${ }^{49}$ Another study revealed that with the stimulation of osteogenic transcription factors, the $\mathrm{Wnt} / \beta$-catenin signaling pathway also led the PDL fibroblasts differentiated into the osteogenic lineage. ${ }^{50}$

To further investigated whether AuNPs and hBD3 promoted osteogenesis via the $\mathrm{Wnt} / \beta$-catenin signaling pathway, ICG-001, a specific inhibitor of $\mathrm{Wnt} / \beta$-catenin signaling pathway, was applied. ICG-001, a novel small molecule inhibitor of $\mathrm{T}$-cell factor/ $\beta$-catenin-dependent transcription, resulted in the downregulation of a subset of target genes that had been implicated in fibrosis. ${ }^{51}$ In our study, ICG-001 reduced the levels of $\beta$-catenin and subsequently depressed the transactivation of the target gene cyclin D1. We also found that hPDLCs treated with ICG-001 reversed the AuNPs + hBD3 + E. coli-LPS-stimulated ALP activity and the formation of calcium deposition. The positive role of AuNPs + hBD3+E. coli-LPS on the mRNA and protein levels of ALP, COL-1, and Runx-2 was also partially blocked by the ICG-001 inhibition of the Wnt/ $\beta$-catenin signaling pathway. These findings revealed that AuNPs + hBD3 + E. coli-LPS upregulated the expression of osteogenic markers via activating the $\mathrm{Wnt} / \beta$-catenin signaling pathway.

There were still several limitations in this study. First, hPDLCs formed a mixed population with limited differentiation potential as they contained a high proportion of terminally differentiated cells. However, PDL stem cells (PDLSCs), which were separated from PDLCs, had exhibited stronger osteogenic differentiation potentials than PDLCs and might be the better candidates for the study of periodontal regeneration. Furthermore, the effect of AuNPs and hBD3 to repair larger periodontal defects induced by periodontal disease should be further validated in vivo. Thus, more researches are needed to confirm these conclusions.

\section{Conclusion}

This study demonstrated that hBD3-combined AuNPs could significantly promote the osteogenic differentiation of hPDLCs in inflammatory microenvironments via activating the $\mathrm{Wnt} / \beta$-catenin signaling pathway. Based on these findings, we had reasons to believe that hBD3-combined AuNPs have the potential to be applied in periodontal local delivery systems, which could deliver the drug to the periodontal pocket and surrounding tissues to promote the biology of wound healing of periodontal defects in the future. Furthermore, hBD3-combined AuNPs might be the attractive and promising candidates for bone formation and periodontal regeneration in periodontitis.

\section{Acknowledgments}

This study was supported by the National Natural Science Foundation Project (No 81570982), the International Cooperation Research and Develop Project of Nanjing Health Bureau (No 201605083), and the Project of Invigorating Health Care through Science, Technology and Education (No CXTDB2017014). The authors thank Central Laboratory of Stomatology, Nanjing Stomatological Hospital, Medical School of Nanjing University.

\section{Disclosure}

The authors report no conflicts of interest in this work.

\section{References}

1. Hajishengallis G. Periodontitis: from microbial immune subversion to systemic inflammation. Nat Rev Immunol. 2015;15(1):30-44.

2. Alvarez R, Lee HL, Wang CY, Hong C. Characterization of the osteogenic potential of mesenchymal stem cells from human periodontal ligament based on cell surface markers. Int J Oral Sci. 2015;7(4): 213-219.

3. Khan SA, Kong EF, Meiller TF, Jabra-Rizk MA. Periodontal diseases: bug induced, host promoted. PLoS Pathog. 2015;11(7):e1004952.

4. Hughes FJ, Ghuman M, Talal A. Periodontal regeneration: a challenge for the tissue engineer? Proc Inst Mech Eng H. 2010;224(12):1345-1358.

5. Kato H, Taguchi Y, Tominaga K, Umeda M, Tanaka A. Porphyromonas gingivalis LPS inhibits osteoblastic differentiation and promotes pro-inflammatory cytokine production in human periodontal ligament stem cells. Arch Oral Biol. 2014;59(2):167-175.

6. Gorr SU. Antimicrobial peptides in periodontal innate defense. Front Oral Biol. 2012;15:84-98.

7. Gomes Pde S, Fernandes MH. Defensins in the oral cavity: distribution and biological role. J Oral Pathol Med. 2010;39(1):1-9.

8. Bian T, Li L, Lyu J, Cui D, Lei L, Yan F. Human beta-defensin 3 suppresses Porphyromonas gingivalis lipopolysaccharide-induced inflammation in RAW 264.7 cells and aortas of ApoE-deficient mice. Peptides. 2016;82:92-100.

9. Bian T, Li H, Zhou Q, Ni C, Zhang Y, Yan F. Human beta-defensin 3 reduces TNF-alpha-induced inflammation and monocyte adhesion in human umbilical vein endothelial cells. Mediators Inflamm. 2017; 2017:8529542.

10. Lyu J, Bian T, Chen B, et al. Beta-defensin 3 modulates macrophage activation and orientation during acute inflammatory response to Porphyromonas gingivalis lipopolysaccharide. Cytokine. 2017;92:48-54.

11. Kraus D, Deschner J, Jager A, et al. Human beta-defensins differently affect proliferation, differentiation, and mineralization of osteoblast-like MG63 cells. J Cell Physiol. 2012;227(3):994-1003.

12. Wang $\mathrm{H}$, Watanabe $\mathrm{H}$, Ogita $\mathrm{M}$, Ichinose S, Izumi Y. Effect of human beta-defensin-3 on the proliferation of fibroblasts on periodontally involved root surfaces. Peptides. 2011;32(5):888-894.

13. Heo DN, Yang DH, Moon HJ, et al. Gold nanoparticles surfacefunctionalized with paclitaxel drug and biotin receptor as theranostic agents for cancer therapy. Biomaterials. 2012;33(3):856-866.

14. Dykman L, Khlebtsov N. Gold nanoparticles in biomedical applications: recent advances and perspectives. Chem Soc Rev. 2012;41(6): 2256-2282. 
15. Jahangirian H, Lemraski EG, Webster TJ, Rafiee-Moghaddam R, Abdollahi Y. A review of drug delivery systems based on nanotechnology and green chemistry: green nanomedicine. Int J Nanomedicine. 2017;12:2957-2978.

16. Ko WK, Heo DN, Moon HJ, et al. The effect of gold nanoparticle size on osteogenic differentiation of adipose-derived stem cells. J Colloid Interface Sci. 2015;438:68-76.

17. Yi CQ, Liu DD, Fong CC, Zhang JC, Yang MS. Gold nanoparticles promote osteogenic differentiation of mesenchymal stem cells through p38 MAPK pathway. ACS Nano. 2010;4(11):6439-6448.

18. Liu D, Zhang J, Yi C, Yang M. The effects of gold nanoparticles on the proliferation, differentiation, and mineralization function of MC3T3-E1 cells in vitro. Chin Sci Bull. 2010;55(11):1013-1019.

19. Rau LR, Huang WY, Liaw JW, Tsai SW. Photothermal effects of laser-activated surface plasmonic gold nanoparticles on the apoptosis and osteogenesis of osteoblast-like cells. Int J Nanomedicine. 2016;11: 3461-3473.

20. Li J, Li JJ, Zhang J, Wang X, Kawazoe N, Chen G. Gold nanoparticle size and shape influence on osteogenesis of mesenchymal stem cells. Nanoscale. 2016;8(15):7992-8007.

21. Jiang W, Kim BY, Rutka JT, Chan WC. Nanoparticle-mediated cellular response is size-dependent. Nat Nanotechnol. 2008;3(3):145-150.

22. Zhang Y, Kong N, Zhang Y, Yang W, Yan F. Size-dependent effects of gold nanoparticles on osteogenic differentiation of human periodontal ligament progenitor cells. Theranostics. 2017;7(5):1214-1224.

23. Rossini M, Gatti D, Adami S. Involvement of WNT/beta-catenin signaling in the treatment of osteoporosis. Calcif Tissue Int. 2013; 93(2):121-132.

24. Choi SY, Song MS, Ryu PD, Lam AT, Joo SW, Lee SY. Gold nanoparticles promote osteogenic differentiation in human adipose-derived mesenchymal stem cells through the Wnt/beta-catenin signaling pathway. Int J Nanomedicine. 2015;10:4383-4392.

25. Saidak Z, Le Henaff C, Azzi S, et al. Wnt/beta-catenin signaling mediates osteoblast differentiation triggered by peptide-induced alpha5beta1 integrin priming in mesenchymal skeletal cells. J Biol Chem. 2015;290(11):6903-6912.

26. Zhang Y, Liu J, Li D, et al. Self-assembled core-satellite gold nanoparticle networks for ultrasensitive detection of chiral molecules by recognition tunneling current. ACS Nano. 2016;10(5):5096-5103.

27. Wolf M, Lossdorfer S, Abuduwali N, et al. In vivo differentiation of human periodontal ligament cells leads to formation of dental hard tissue. J Orofac Orthop. 2013;74(6):494-505.

28. Berahim Z, Moharamzadeh K, Jowett AK, Rawlinson A. Evaluation of osteogenic and cementogenic potential of periodontal ligament fibroblast spheroids using a three-dimensional in vitro model of periodontium. Int J Dent. 2015;2015:605813.

29. Wu C, Zhou Y, Lin C, Chang J, Xiao Y. Strontium-containing mesoporous bioactive glass scaffolds with improved osteogenic/cementogenic differentiation of periodontal ligament cells for periodontal tissue engineering. Acta Biomater. 2012;8(10):3805-3815.

30. Xia Y, Tang HN, Wu RX, Yu Y, Gao LN, Chen FM. Cell responses to conditioned media produced by patient-matched stem cells derived from healthy and inflamed periodontal ligament tissues. J Periodontol. 2016;87(5):e53-e63.

31. Chen G, Goeddel DV. TNF-R1 signaling: a beautiful pathway. Science. 2002;296(5573):1634-1635.

32. Lee DC, Lau AS. Effects of Panax ginseng on tumor necrosis factor-alpha-mediated inflammation: a mini-review. Molecules. 2011; 16(4):2802-2816.

33. Albiero ML, Amorim BR, Casati MZ, Sallum EA, Nociti FHJ, Silverio KG. Osteogenic potential of periodontal ligament stem cells are unaffected after exposure to lipopolysaccharides. Braz Oral Res. 2017;31:e17.
34. Huang TH, Chen CC, Liu SL, Lu YC, Kao CT. A low-level diode laser therapy reduces the lipopolysaccharide (LPS)-induced periodontal ligament cell inflammation. Laser Phys Lett. 2014;11:1-7.

35. Alkilany AM, Murphy CJ. Toxicity and cellular uptake of gold nanoparticles: what we have learned so far? J Nanopart Res. 2010;12(7): 2313-2333.

36. Chithrani BD, Ghazani AA, Chan WCW. Determining the size and shape dependence of gold nanoparticle uptake into mammalian cells. Nano Lett. 2006;6(4):662-668.

37. Chithrani BD, Chan WCW. Elucidating the mechanism of cellular uptake and removal of protein-coated gold nanoparticles of different sizes and shapes. Nano Lett. 2007;7(6):1542-1550.

38. Ravichandran R, Venugopal JR, Sundarrajan S, Mukherjee S, Ramakrishna S. Precipitation of nanohydroxyapatite on PLLA/PBLG/ Collagen nanofibrous structures for the differentiation of adipose derived stem cells to osteogenic lineage. Biomaterials. 2012;33(3):846-855.

39. Ravikumar K, Boda SK, Basu B. Synergy of substrate conductivity and intermittent electrical stimulation towards osteogenic differentiation of human mesenchymal stem cells. Bioelectrochemistry. 2017;116:52-64.

40. Liu DD, Yi CQ, Zhang DW, Zhang JC, Yang MS. Inhibition of proliferation and differentiation of mesenchymal stern cells by carboxylated carbon nanotubes. ACS Nano. 2010;4(4):2185-2195.

41. Sengupta P, Xu Y, Wang L, Widom R, Smith BD. Collagen alpha1(I) gene (COL1A1) is repressed by RFX family. J Biol Chem. 2005; 280(22):21004-21014.

42. Albiero ML, Stipp RN, Saito MT, et al. Viability and osteogenic differentiation of human periodontal ligament progenitor cells are maintained after incubation with Porphyromonas gingivalis protein extract. J Periodontol. 2017;88(11):e188-e199.

43. Liu H, Peng H, Wu Y, et al. The promotion of bone regeneration by nanofibrous hydroxyapatite/chitosan scaffolds by effects on integrinBMP/Smad signaling pathway in BMSCs. Biomaterials. 2013;34(18): 4404-4417.

44. Zhang D, Liu D, Zhang J, Fong C, Yang M. Gold nanoparticles stimulate differentiation and mineralization of primary osteoblasts through the ERK/MAPK signaling pathway. Mater Sci Eng C Mater Biol Appl. 2014;42:70-77.

45. Taton TA. Nanotechnology - boning up on biology. Nature. 2001; 412(6846):491-492.

46. Varoga D, Wruck CJ, Tohidnezhad M, et al. Osteoblasts participate in the innate immunity of the bone by producing human beta defensin-3. Histochem Cell Biol. 2009;131(2):207-218.

47. Winter J, Pantelis A, Reich R, et al. Human beta-defensin-1, -2, and -3 exhibit opposite effects on oral squamous cell carcinoma cell proliferation. Cancer Invest. 2011;29(3):196-201.

48. Ferris LK, Mburu YK, Mathers AR, et al. Human beta-defensin 3 induces maturation of human langerhans cell-like dendritic cells: an antimicrobial peptide that functions as an endogenous adjuvant. J Invest Dermatol. 2013;133(2):460-468.

49. Premaraj S, Souza I, Premaraj T. Mechanical loading activates betacatenin signaling in periodontal ligament cells. Angle Orthod. 2011; 81(4):592-599.

50. Heo JS, Lee SY, Lee JC. Wnt/beta-catenin signaling enhances osteoblastogenic differentiation from human periodontal ligament fibroblasts. Mol Cells. 2010;30(5):449-454.

51. Henderson WR Jr, Chi EY, Ye X, et al. Inhibition of Wnt/beta-catenin/ CREB binding protein (CBP) signaling reverses pulmonary fibrosis. Proc Natl Acad Sci US A. 2010;107(32):14309-14314. 
International Journal of Nanomedicine

Dovepress

\section{Publish your work in this journal}

The International Journal of Nanomedicine is an international, peerreviewed journal focusing on the application of nanotechnology in diagnostics, therapeutics, and drug delivery systems throughout the biomedical field. This journal is indexed on PubMed Central, MedLine, CAS, SciSearch ${ }^{\circledR}$, Current Contents ${ }^{\circledR} /$ Clinical Medicine,
Journal Citation Reports/Science Edition, EMBase, Scopus and the Elsevier Bibliographic databases. The manuscript management system is completely online and includes a very quick and fair peer-review system, which is all easy to use. Visit http://www.dovepress.com/ testimonials.php to read real quotes from published authors.

Submit your manuscript here: http://www.dovepress.com/international-journal-of-nanomedicine-journal 